\title{
COMPOSTOS ORGANO-HALOGENADOS ADSORVÍVEIS (AOX) NA ÁGUA DE ABASTECIMENTO PÚBLICO DOS MUNICÍPIOS DE ITAJAÍ E NAVEGANTES - SC
}

\author{
DUARTE, A.C.E.; RÖRIG, L.R.*; AMARAL, M.D.; VIEIRA, M.G.V.; DADAM, L. \\ Centro de Ciências Tecnológicas da Terra e do Mar (CTTMar). Universidade do Vale do Itajaí \\ (UNIVALI) - Rua Uruguai, 485 - Centro - Itajaí - SC - Brasil - CEP 88.302-020. \\ *Corresponding author: rorig@univali.br
}

\begin{abstract}
Duarte, A.C.E.; Rörig, L.R.; Amaral, M.D.; Vieira, M.G.V.; Dadam, L. 2009. Absorbable Organic Halogens (AOX) in the Public Water Supply of the Municipalities of Itajaí and Navegantes - SC. Braz. J. Aquat. Sci. Technol. 13(1): 11-17. ISSN 1808-7035. Absorbable Organic Halogens (AOX) include a number of substances resulting from the reaction of halogens (fluorine, chlorine, bromine and iodine) with organic molecules, being mainly produced by anthropogenic activities. Their considerable toxicity and environmental persistence demand monitoring in surface waters and effluents. AOX are produced as waste from industrial activities such as chemical washing of clothes, metal degreasing, paper bleaching and water treatment with chlorine compounds. Also, AOX wastes are produced by domestic and agricultural activities, by using disinfectants and pesticides. Once AOX is a sum parameter, it does not discriminate which specific molecules are being analyzed, but it is a good parameter to use in preliminary evaluations of water and effluent quality. This paper presents the results of a monitoring initiative of AOX in public water supply in Southern Brazil. The study area included a region which has considerable impacts on its water resources as a consequence of domestic and industrial pollution and land misuse. The results showed high levels of AOX in treated water (overall average $=0.26 \mathrm{mg}^{-\mathrm{L}^{-1}}$ ), which were higher than those found in surface waters (general media $\left.=0.028 \mathrm{mg} \cdot \mathrm{L}^{-1}\right)$, indicating that the formation of these toxic compounds is related especially with the chlorination of water in the process of treatment. Probably, the poor quality of raw water allows the persistence of dissolved organic matter even after the water treatment, when chlorine disinfection resulted in AOX production. Concentrations of AOX in the public water supply indicate health risks to human consumers and environment.
\end{abstract}

Keywords: water pollution; AOX; persistent organic pollutants; Itajaí-Mirim river; water treatment and supply.

\section{INTRODUÇÃO}

Os compostos organo-halogenados são principalmente de origem industrial (Cherif et al., 2006), apesar de também existirem organo-halogenados de origem natural (Asplund \& Grimvall, 1991). Esses compostos incluem uma grande quantidade de moléculas diferentes cuja análise qualitativa individual é cara e trabalhosa. Em razão disso são geralmente analisados na forma de parâmetros de soma, sendo a concentração de organo-halogenados adsorvíveis (AOX) um dos mais comumente utilizados em análises de monitoramento ambiental (Müller, 2003). O grupo AOX abrange muitos compostos do tipo pesticida, plásticos, solventes, lubrificantes e medicamentos, incluindo moléculas de grande toxicidade, persistência e bioacumulação em águas, plantas e animais como os PCBs (bifenilas policloradas), as dioxinas e o HCB (hexaclorobenzeno) ou dieldrin (Cherif et al., 2006).

A produção de compostos $A O X$ cresceu muito no século 20 devido às suas propriedades químicas que possibilitam aplicações como desinfetantes, biocidas, agentes de limpeza, solventes, extintores e refrigerantes industriais. Indústrias de celulose e papel, de tratamento de superfícies de metais, curtumes, lavanderias a seco entre outras são importantes geradoras de AOX em seus efluentes, mas efluentes domésticos como lixo e esgoto urbano também contribuem significativamente (Polkowska, 2003). Além disso, a cloração de águas em estações de tratamento ou mesmo em piscinas pode produzir AOX em vias que podem atingir seres humanos rapidamente e diretamente (Tominaga \& Midio, 1999). Freqüentemente, os compostos organo-halogenados mais encontrados são os organoclorados com um, dois ou mais átomos de carbono (Polkowska, 2003).

Entre os efeitos gerados pelos organohalogenados diversos estudos apontam o desenvolvimento de neoplasias no homem, efeitos na reprodução e desenvolvimento embrionário, provocando aumento significativo na incidência de abortos e natimortos, possível envolvimento com a esterilidade, efeitos 

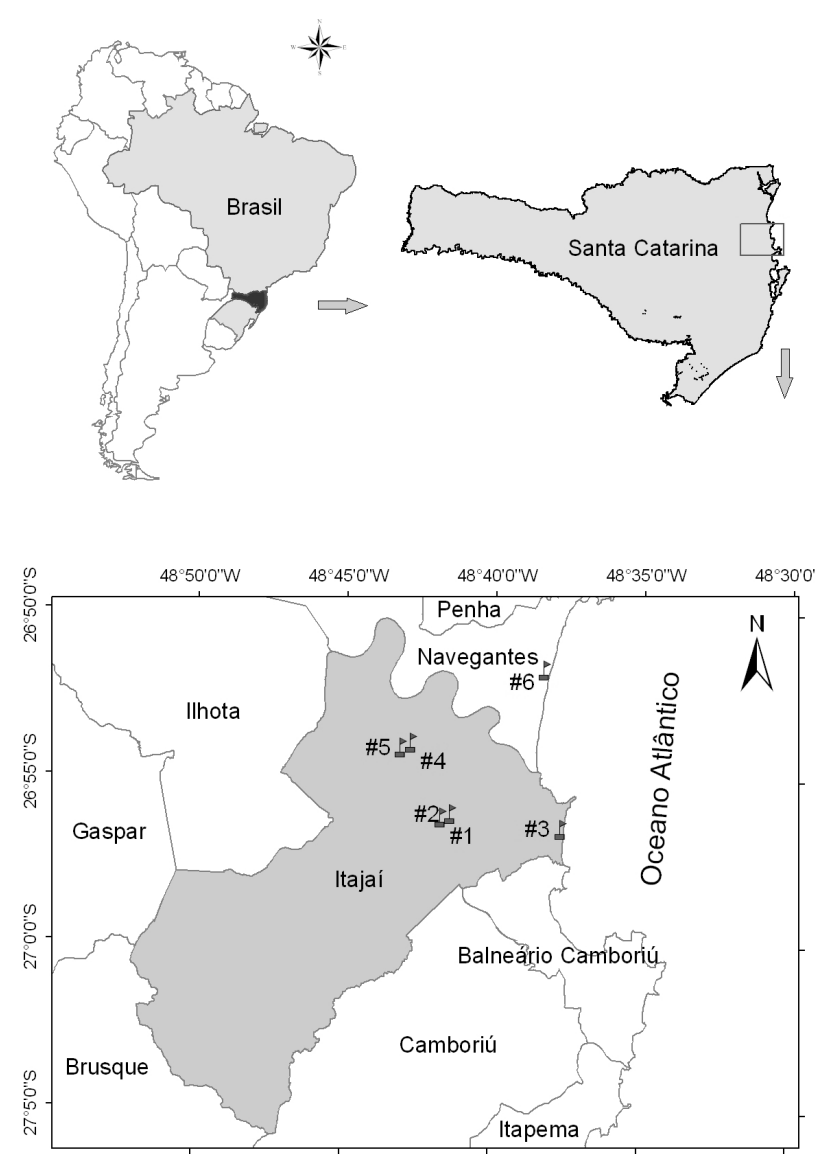

rigura 1 - Area de estudo com a disposiçäo das estaçöes amostrais. Localização das estações: \#1: captação do rio Canhanduba; \#2: início da rede Canhanduba, bairro Canhanduba - Itajaí; \#3: ponta da rede Canhanduba, bairro Praia Brava - Itajaí; \#4: captação do rio Itajaí-Mirim; \#5: início da rede Itajaí-Mirim, bairro São Roque Itajaí; \#6: ponta da rede Itajaí-Mirim, município de Navegantes.

estrogênicos fracos que podem precipitar o parto, aumento na incidência de câncer do endotélio e da mama, decréscimo na qualidade do sêmen, alteração no trato reprodutivo masculino, aumento da incidência de câncer testicular e disrupção endócrina (Longnecker et al. 1997; Shukla et al., 2001; Delgado et al., 2002). Agem também sobre o sistema nervoso central, diminuindo a velocidade de condução em nervos motores e atuando na degeneração neuronal que leva à doença de Parkinson (Nunes \& Tajara, 1998; Tominaga \& Midio, 1999).

A relação entre o uso de cloro nas estações de tratamento de água, suas reações com os compostos orgânicos presentes e a formação de compostos que poderiam ter efeito negativo sobre a saúde humana foi estudada pela primeira vez por R. H. Harris, na década de 1970 (Santos, 1987). A partir de suas indicações pioneiras sobre a possibilidade de existir uma correlação entre águas de abastecimento e câncer, outros pes- quisadores passaram a se interessar pelo assunto (Meyer, 1994).

No Brasil, a Portaria ํo 518 de 25/03/2004 do Ministério da Saúde estabelece os procedimentos e responsabilidades relativas ao controle e vigilância, bem como o limite máximo permissível de $0,1 \mathrm{mg} \cdot \mathrm{L}^{-1}$, para a soma das concentrações de trialometanos (TAM; clorofórmio ou triclorometano + bromodiclorometano + dibromoclorometano + bromofórmio ou tribromometano) na água para consumo humano. No Canadá o limite é de $0,350 \mathrm{mg} \cdot \mathrm{L}^{-1}$, na Alemanha $0,025 \mathrm{mg} \cdot \mathrm{L}^{-1}$, na Holanda 0,075 mg. L-1 e na França 0,010 g. L-1 (Meyer, 1994).

Esse trabalho teve por objetivo avaliar os teores de AOX nas águas brutas e tratadas para o abastecimento nos municípios de Itajaí e Navegantes (SC Brasil) para gerar um panorama de risco sanitário associado à poluição dos mananciais e aos efeitos do processo de cloração.

\section{MATERIAL E MÉTODOS}

\section{Área de Estudo}

A área de estudo desse trabalho abrange o sistema de captação e distribuição de água para o abastecimento público dos municípios de Itajaí e Navegantes (SC - Brasil), administrado pelo Serviço Municipal de Água e Saneamento de Itajaí (SEMASA). Os locais de captação (água bruta) localizam-se nos rios Itajaí-Mirim e Canhanduba. Em relação ao sistema de distribuição de água tratada, optou-se por definir um ponto no início e outro na ponta de distribuição de cada rede. A Estação de Tratamento de Água do rio Itajaí-Mirim (ETA São Roque) produz 612 L.s. ${ }^{-1}$ e a ETA do rio Canhanduba (ETAArapongas) produz 140 L.S $^{-1}$.

As estações amostrais \#1 e \#4 correspondem aos pontos de captação de água bruta dos rios Canhanduba e rio Itajaí-Mirim, respectivamente. As estações \#2 (bairro Canhanduba) e \#3 (bairro Praia Brava) representam, respectivamente, o início e ponta de rede de abastecimento das águas tratadas do rio Canhanduba. As estações \#5 (bairro São Roque) e \#6 (município de Navegantes) representam, respectivamente, o início e ponta de rede das águas tratadas do rio Itajaí-Mirim (Figura 1). Essa estratégia amostral teve o objetivo de avaliar a concentração de AOX nos corpos receptores e sua produção imediata (início de rede) ou retardada (ponta de rede) no sistema de abastecimento.

O período amostral foi compreendido entre novembro de 2004 e julho de 2005, com um total de 15 amostragens em intervalos de 15 dias. 


\section{Análises de água}

As variáveis físico-químicas oxigênio dissolvido (OD), $\mathrm{pH}$, temperatura, turbidez e condutividade elétrica foram analisadas in situ ou imediatamente após a coleta, tendo-se o cuidado de não gerar oxigenação mecânica. As análises foram realizadas com um multianalisador Horiba ${ }^{\circledR}$ U10.

Alíquotas das amostras de água foram separadas para a determinação de AOX (organo-halogenados adsorvíveis), as quais foram acondicionadas em garrafas plásticas tipo PET de $500 \mathrm{~mL}$ previamente lavadas com detergente EXTRAN ${ }^{\circledR}$, depois com solução de ácido nítrico a $2 \%$ e em seguida enxaguadas com água destilada. As amostras eram acidificadas com 5-7 gotas de ácido nítrico concentrado para que o $\mathrm{pH}$ ficasse abaixo de 2, sendo mantidas refrigeradas em isopor com gelo até a chegada ao laboratório para processamento.

As determinações de AOX seguiram as recomendações de normativas européias (ISO/CEI/EN 17025, 2005; EN 1485, 1996; DIN 38409-H14-H14. 1985; Ströhlein GmbH \& CO, 1996). Para as análises, $100 \mathrm{ml}$ de amostra tiveram o $\mathrm{pH}$ reduzido para um valor entre 0 e 2 com ácido nítrico concentrado (2-3 gotas), sendo adicionadas de $5 \mathrm{ml}$ de nitrato de sódio $0,2 \mathrm{M}$ e de 65 mg de carvão ativado para AOX (MERCK ${ }^{\circledR}$, granulometria $<150 \mathrm{~mm}$, superfície específica mínima de $850 \mathrm{~m}^{2} \cdot \mathrm{g}^{-1}$ ). As amostras foram agitadas por 2 horas e então filtradas através de membrana de policarbonato (porosidade de $0,2 \mu \mathrm{m}$ ). Cada membrana com o material retido (carvão ativado + compostos adsorvidos) foi colocada no forno do analisador de AOX Coulomat ${ }^{\circledR} 7020 \mathrm{CL}$ para combustão a $950^{\circ} \mathrm{C}$. No processo, um fluxo de oxigênio analítico 8.0 arrasta os gases primeiramente até a unidade de secagem, contendo ácido sulfúrico concentrado, onde ocorre o resfriamento dos gases. Após passar pela unidade de secagem, os gases, chegam à célula eletrolítica, onde estão eletrodos de prata e platina e também a cadeia de medição, onde ocorre a reação entre os halogênios, oriundos da combustão do filtrado, e a prata liberada pelo eletrodo. A cadeia de medição quantifica esta reação entre halogênios e prata. Os resultados foram expressos em mg Cl.100 mL-1 e então convertidos para mg. $\mathrm{L}^{-1}$.

Dados de cloro residual foram gentilmente fornecidos pelo SEMASA, no entanto esses dados são de análises feitas na saída das Estações de Tratamento de Água (ETAs) e não das estações de coleta das demais variáveis. Tais dados foram obtidos a fim de relacioná-los com os valores de AOX das estações de início de rede (estações \#2 e \#5).

\section{RESULTADOS E DISCUSSÃO}

\section{Variáveis físico-químicas e cloro residual}

Os valores de temperatura da água foram similares entre as diversas estações considerando-se os mesmos dias de coleta. A variação está relacionada à temperatura do ar que segue um padrão sazonal. $\mathrm{O}$ valor médio maior para a estação \#3 deve estar relacionado à distância desse ponto (ponta de rede), o que determina maior aquecimento solar em seu percurso (Figura 2).

A condutividade mostrou valores médios mais elevados nas amostras relacionadas ao rio Itajaí-Mirim (estações \#4, \#5 e \#6; Figura 2). Isso se deve provavelmente à influência da salinidade marinha no ponto de captação devido ao efeito da progressão da cunha salina. Sais não removidos no processo de tratamento, bem como os sais adicionados ou gerados no processo de tratamento de água (sulfato de alumínio, hipoclorito de sódio, fluor-silicatos, cal e possíveis derivados e contaminantes) também influenciam a condutividade de águas de abastecimento já que podem permanecer como resíduos na água tratada (Drinan, 2000).

$\mathrm{O} \mathrm{pH}$ mostrou valores médios levemente ácidos (Figura 2). As águas tratadas mostraram valores mais altos que as águas naturais (mananciais). Provavelmente isso se deve a neutralização da água com cal após o tratamento.

Segundo a Resolução CONAMA no 357 de 17 de Março de 2005, para águas doces de classe II, o limite máximo permitido de turbidez é de 100 UNT. As médias das estações \#1 e \#4 mantiveram-se dentro dos limites dessa Resolução, porém, na estação \#4 em algumas datas foram registrados valores superiores a 100 UNT (Figura 2). As variações de turbidez nesses locais junto aos pontos de captação de água para abastecimento estão relacionadas a eventos de chuva, que por sua vez exigem aumento no uso de coagulantes e na intensidade de cloração. Essas estações estão sob influência de culturas agrícolas, principalmente de arroz irrigado, que na região contribui intensamente para o aumento da turbidez e material particulado em suspensão (Rörig, 2005; Pereira-Filho, 2006). Outro fator a ser levado em consideração em relação à turbidez é o escoamento do material sedimentado nos decantadores das ETAs que são lançados próximos aos pontos de captação. No caso dessas estações a existência de efeito de maré pode deslocar a pluma desses resíduos para montante, onde podem novamente ser captados pelo bombeamento das ETAs. A construção recente de uma barragem junto a estação \#4 e de um represamento precário próximo da estação \#1 deve amenizar ou solucionar os problemas relacionados à maré. Nas amos- 
Temperatura $\left({ }^{\circ} \mathrm{C}\right)$
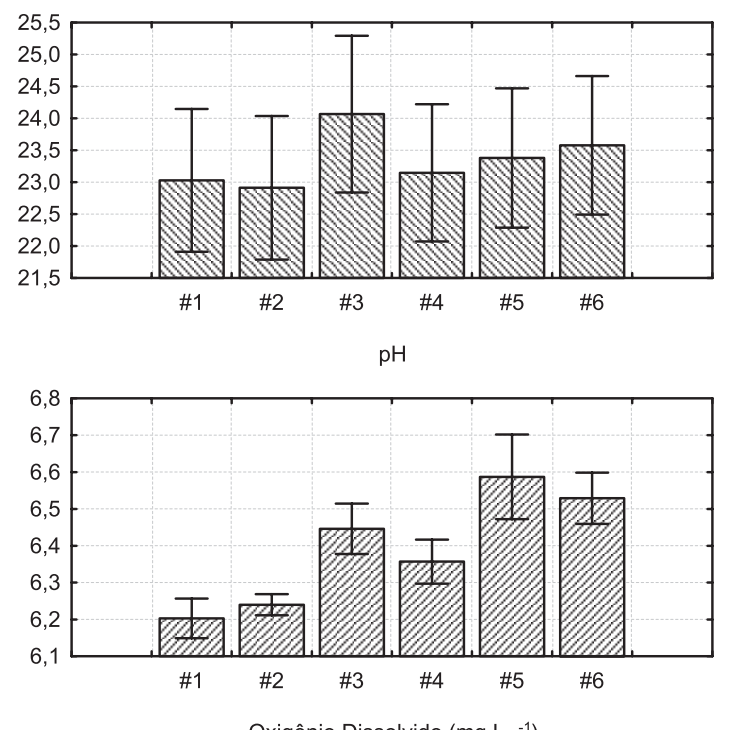

Oxigênio Dissolvido (mg.L ${ }^{-1}$ )

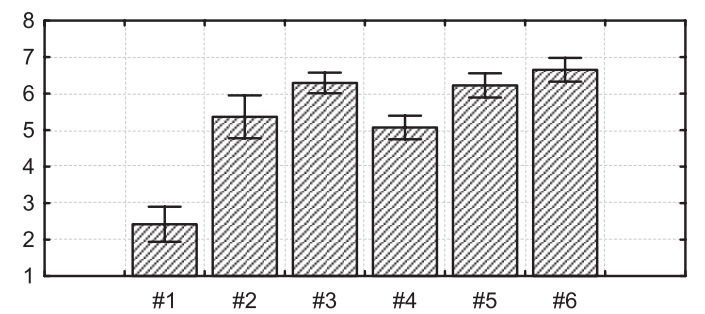

Condutividade $\left(\mathrm{mS}_{\mathrm{cm}}{ }^{-1}\right)$
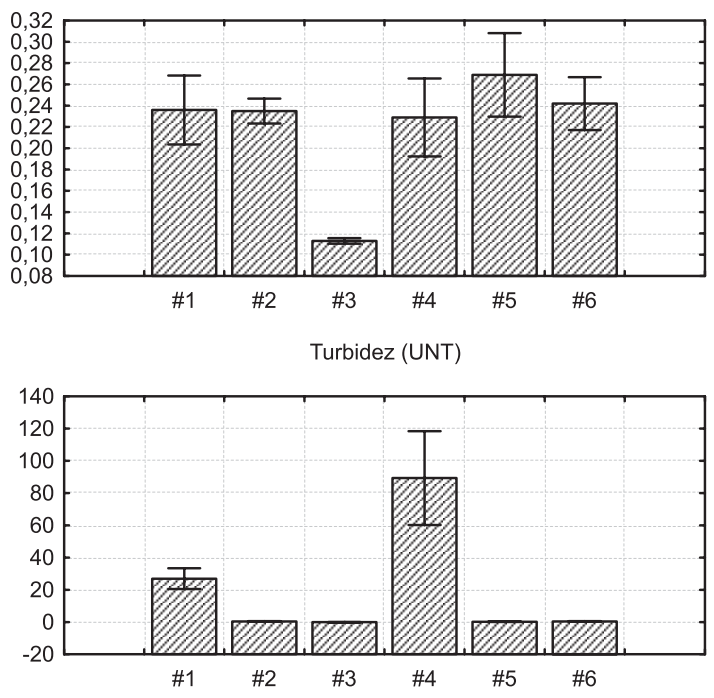

Cloro residual (mg. $\left.\mathrm{L}^{-1}\right)$

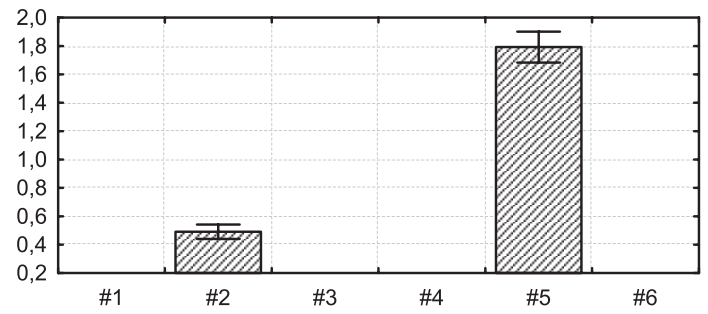

Figura 2 - Valores médios das variáveis físico-químicas e cloro residual durante o período amostral ( $n=15)$. As barras indicam o erro padrão da média. Estações: \#1 - captação do rio Canhanduba; \#2 - início da rede Canhanduba; \#3 - ponta da rede Canhanduba; \#4 captação do rio Itajaí-Mirim; \#5 - início da rede Itajaí-Mirim; \#6 - ponta da rede Itajaí-Mirim.

tras de água tratada (estações \#2, \#3, \#5 e \#6) a turbidez foi indetectável (Figura 2).

Em relação ao oxigênio dissolvido, que nas estações fluviais (\#1 e \#4) não pode ser inferior a $5 \mathrm{mg} \cdot \mathrm{L}^{-1}$, houve várias desconformidades com a Resolução CONAMA n 357 . A média para a estação \#1 foi de 2,4 $\mathrm{mg} \cdot \mathrm{L}^{-1}$, o que sugere a inadequação do uso dessas águas para abastecimento público, mesmo considerando uma boa eficiência de tratamento nas ETAs. Águas com valores baixos de oxigênio dissolvido, em geral são fortemente poluídas com matéria orgânica. Em função disso, a eficiência do tratamento diminui e há mais riscos de contaminação microbiológica ou de formação de compostos tóxicos quando da pré-cloração que eventualmente pode ser realizada nas ETAs em questão. Acredita-se que existam três fontes de poluição que podem estar contribuindo para o consumo de oxigênio dissolvido nas águas do rio Canhanduba (estação \#1): os resíduos da rizicultura irrigada, já que este rio corta extensas áreas dessa cultura; a descarga em local à montante da estação \#1 de efluente tratado de chorume oriundo de aterro sanitário; e ainda os lançamentos de esgoto in natura por populações ribeirinhas próximas à essa estação. A estação do rio ItajaíMirim (\#4) apresentou média de oxigênio dissolvido le- vemente superior ao limite mínimo estabelecido, porém em várias situações os valores foram inferiores ao mesmo, evidenciando também comprometimento desse recurso hídrico. As razões prováveis são similares ao caso do rio Canhanduba, exceto para o caso dos resíduos de chorume. Entretanto, o rio Itajaí-Mirim recebe uma forte carga poluidora representada por efluentes industriais e pelos esgotos não tratados do município de Brusque e também dos bairros ribeirinhos em Itajaí. Vários estudos regionais já mostraram que esse rio representa um dos piores recursos hídricos de toda a Bacia do Itajaí em termos de qualidade geral de suas águas (Miranda et al. 2003; Ferreira \& Beaumord, 2004; Rörig, 2005; Burliga et al., 2005; Pereira-Filho, 2006).

Os dados de cloro residual foram apresentados como sendo das estações \#2 e \#5, pois estas se localizam próximas, respectivamente, às ETAs do rio Canhanduba e do Rio Itajaí-Mirim. Os valores foram sensivelmente maiores para a estação \#5, indicando possivelmente uma cloração mais intensa nessa ETA, já que a água produzida na mesma percorre uma rede de distribuição mais longa, além de a produção de água ser maior. 


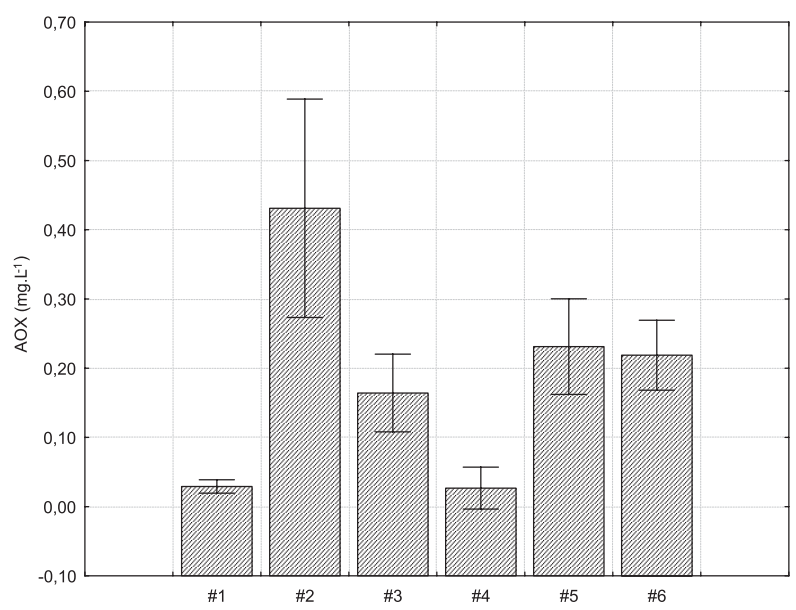

Figura 3 - Valores médios de organo-halogenados adsorvíveis (AOX) para o período amostral $(n=15)$. As barras indicam o desvio padrão da média. Estações: \#1 - captação do rio Canhanduba; \#2 - início da rede Canhanduba; \#3 - ponta da rede Canhanduba; \#4 - captação do rio Itajaí-Mirim; \#5 - início da rede Itajaí-Mirim; \#6 ponta da rede Itajaí-Mirim.

\section{AOX}

Os valores de AOX mostraram claramente um aumento nas águas tratadas (estações \#2, \#3, \#5 e \#6) quando comparados às águas superficiais (estações \#1 e \#4; Figura 3). Nas estações de águas superficiais os valores foram baixos e com pequena variação em torno da média. As maiores médias foram obtidas nas estações de início de rede de distribuição, especialmente na estação \#2, correspondente à água tratada do rio Canhanduba. $O$ fato de os valores terem sido maiores no início de rede (estações \#2 e \#5) pode estar relacionado à maior concentração de cloro nesses pontos, incrementando a formação de AOX, e também ao decaimento desses compostos nas pontas de rede em função de volatilização, degradação e adsorção. A pequena diferença entre as médias das estações \#5 e \#6 deve estar relacionada ao fato de que a estação \#6, apesar de distante da estação de tratamento, recebe água por uma tubulação linear, sem ramificações, o que acaba encurtando o trajeto da água. Tal informação foi obtida somente após o término das amostragens, por consulta à empresa de tratamento de água (SEMASA - Serviço Municipal de Água, Saneamento Básico e Infra-estrutura).

Em análises exploratórias de correlação entre as variáveis físico-químicas e o AOX não foi constatada qualquer relação significativa de dependência. Através de análise de correlação utilizando software MVSP, verificou-se para as estações \#2 e \#5 (inícios de rede), uma correlação negativa entreAOX e cloro residual $(r=-0,63$; $n=30 ; P<0,05)$. A explicação mais plausível para essa relação é a de que o cloro residual tenha sido consumido pela oxidação de matéria orgânica remanescente após o tratamento de água, o que implicaria em considerar que a formação de AOX ocorre já no processo de tratamento e não tanto na rede de distribuição. De qualquer maneira, a pós-cloração é realizada com a finalidade de que haja cloro reativo até as pontas de rede, 0 que não exclui que o processo de formação de AOX ocorra ao longo de todo o percurso da água tratada. Por outro lado, a relação negativa entre AOX e cloro residual indica que essa última variável não interfere nos resultados analíticos do AOX, como poderia se suspeitar. No processo de análise do AOX o cloro inorgânico é eliminado como interferente através da adição de nitrato de sódio no preparo da amostra, o qual reage com os halogênios formando haletos de sódio, estes não são detectados pela cadeia de medição do analisador (Ströhlein Gmbh \& Co, 1996).

Em estudos pioneiros sobre a presença de AOX na Bacia do rio Itajaí, realizados por Amaral (2005) e Rörig (2005), verificou-se um claro padrão de incremento nos níveis de montante para jusante da bacia, com valores máximos justamente no rio Itajaí-Mirim (média de $0,42 \mathrm{mg} \cdot \mathrm{L}^{-1} ; \mathrm{n}=27$ ), em local correspondente à estação \#4 do presente estudo. Os autores sugerem que esse padrão indica uma possível acumulação de tais compostos tóxicos ao longo da bacia. Seu padrão de incremento parece mais evidente justamente por esses compostos serem mais conservativos e dificilmente degradáveis, sugerindo uma situação de risco para as áreas à jusante da bacia. Em relação aos efluentes gerados na bacia foi verificado que as indústrias químicas são as principais contribuintes, seguidas pelas indústrias pesqueiras, com valores médios de $0,52 \mathrm{mg}^{\mathrm{L}}{ }^{-1} \mathrm{e}$ 0,37 mg. $\mathrm{L}^{-1}$, respectivamente (Rörig, 2005). Essa tipologia industrial é típica dos municípios de Blumenau e Brusque (indústrias químicas) e Itajaí e Navegantes (indústrias pesqueiras), o que reforça a situação de risco para a região do Médio e Baixo Vales do rio Itajaí.

Valores de AOX acima de $0,05 \mathrm{mg} . \mathrm{L}^{-1} \mathrm{em}$ águas superficiais já são suficientes para produzir efeitos tóxicos e mutagênicos (Yamamoto et al., 1992). Como as concentrações encontradas nos mananciais do Vale do Itajaí foram em média 10 vezes maiores que o valor acima apontado, é fundamental que investigações sobre a origem e destino do AOX nas águas da região sejam intensificadas. Não há limites estabelecidos para AOX na legislação brasileira, entretanto a Resolução CONAMA no 357/2005 estabelece limites máximos para alguns compostos organo-halogenados possivelmente presentes nas águas superficiais.

Para as águas tratadas, a portaria $n^{0} 518 / 04$ do Ministério da Saúde determina um limite máximo permitido de $0,1 \mathrm{mg} \cdot \mathrm{L}^{-1}$ para trialometanos, sendo estes os compostos mais comumente encontrados nas águas submetidas a tratamento com cloro. Porém este valor não pode ser utilizado como valor limite para as 
análises de AOX realizadas no presente trabalho, visto que AOX é uma medida usada para estimar a quantidade total de compostos organo-halogenados, sem identificação das moléculas presentes. As normativas européias apontam limites de $0,05 \mathrm{mg} \cdot \mathrm{L}^{-1}$ para AOX em águas para abastecimento público (European Community Directive $\left.n^{\circ} 60,2000\right)$. Os dados encontrados no presente estudo chegam a ser 100 vezes maiores que esse limite, o que indica também uma situação de risco à saúde pública na região. Estudos realizados na Ucrânia também indicaram valores similares aos aqui encontrados (Hoffmann \& Michaylenko, 1996). Entretanto, a maior parte dos países apresenta limites legais para substâncias organocloradas específicas, assim como no Brasil. Vários trabalhos técnicos realizados na Alemanha, Ucrânia e Japão sugerem que a análise de AOX seja realizada como um teste prévio em substituição às análises cromatográficas de moléculas específicas (Hoffmann \& Michaylenko, 1996; Yamamoto et al., 1992). Nesse caso, se os valores de AOX forem relevantes, procede-se na análise cromatográfica para diferenciação qualitativa. Os principais argumentos para esse procedimento relacionamse a praticidade e baixo custo da análise de AOX.

A realidade brasileira aponta também nesse sentido, pois são raras as bacias que dispõem de condições financeiras e técnicas para análises de moléculas poluentes específicas. A definição de um valor limite para AOX como parâmetro de soma de organohalogenados em análises de rotina seria mais factível do que a definição de concentrações de dezenas de substâncias.

\section{REFERÊNCIAS}

Amaral, M.D. 2005. Monitoramento dos Teores de Compostos Organo-halogenados Adsorvíveis (AOX) no Baixo Curso do Rio Itajaí-Açu. Trabalho de Conclusão de Curso Biologia - CTTMar - UNIVALI. 51p.

Asplund, G. \& Grimvall, A. 1991. Organohalogens in nature, more widespread than previously assumed. Environ. Sci. Technol. 25:1346-1350.

Burliga, A.L.; Torgan, L.C.; Nobrega, E.A.; Beaumord, A.C.; Costa, C. O. \& Yamauti, D.V. 2005. Diatomáceas epilíticas do Rio Itajaí-Mirim, Santa Catarina, Brasil. Acta Scientiarum. 27 (4): 425-431.

Cherif, S.; Ben Fradj, R. \& Jrad, A. 2006. Quality of treated wastewater: method validation of $A O X$. Accred. Qual. Assur. 11:632-637.

Delgado, I.F; Barreto, H.H.C; Alleluia, I.B; Baggio, C.A. \& Paumgartten, F.J.R. 2002. Serum levels of organochlorine pesticides and polychlorinated biphenyls among inhabitants of Greater Metropolitan Rio de Janeiro, Brazil. Cadernos de Saúde Pública.18: 519-524.
DIN 38409-H14-H14. 1985. Deutsche Einheitsverfahren zur Wasser-, Abwasser- und SchlammUntersuchung: Bestimmung der adsorbierbaren organisch gebundenen Halogene (AOX). Beuth Verlag, Berlin.

Drinan, J.E. 2000. Water and Wastewater Treatment: A Guide for the Nonengineering Professionals. New York, CRC Press. 358p.

EN 1485. 1996. Water quality - Determination of Adsorbable Organically Bound Halogens (AOX). European Standard. Brussola.

European Community Directive $n^{\circ} 60.2000$. Directive of the European Parliament and of the Council of 23 October 2000 establishing a framework for Community action in the field of water policy. Official Journal of the European Communities - L327, 22.12.2000. $72 \mathrm{p}$.

Ferreira, D.M. \& Beaumord, A.C. 2004. Avaliação rápida de integridade ambiental das sub-bacias do rio Itajaí-Mirim no Município de Brusque, SC. Health And Environmental Journal. 5(2): 21-28.

Hoffmann, M. \& Michaylenko, V. 1996. Chlorination of drinking water and its effects in the Ukrainian capital Kiev. Chemistry and Technology of Water. 16 (5): $472-479$.

ISO/CEI/EN 17025. 2005. General requirements for the competence of calibration and testing laboratories. International Standards for Business, Government and Society.

Longnecker, M.P.; Rogan, W.J. \& Lucier, G. 1997. The human health effects of DDT (dichlorodiphenyltrichloroethane) and PCBs (polychlorinated biphenyls) and an overview of organochlorines in public health. Annual Review of Public Health. 18: 211-244.

Meyer, S.T. 1994. O uso do cloro na desinfecção de águas, a formação de trihalometanos e os riscos potenciais à saúde publica. Cad. Saúde Pública. 10(1): 99-110.

Miranda, A.L.B.; Beaumord, A.C. \& Mello, R.S.P. 2003. Aplicação do índice de especificidade de hábitat em Chlorococcales fitoplanctônicas, como indicador de qualidade ambiental em rios costeiros: Estudo de caso do Rio Itajaí-Mirim, SC, Brasil. Notas Técnicas da Facimar. 7: 109-117.

Müller, G. 2003. Sence or no-sence of the sum parameter for water soluble "adsorbable organic halogen" (AOX) and "adsorbable organic halogen (AOX-S18) for the assessment or organohalogens in sludge and sediments. Chemosphere. 52: 371379.

Nunes, M.V. \& Tajara, E.H. 1998. Efeitos tardios dos praguicidas organoclorados no homem. Rev. Saúde Pública. 32(4): 342-383.

Pereira, J.F. 2006. Dinâmica Biogeoquímica do estuário do Rio Itajaí-Açu, SC. Tese de Doutorado. Pro- 
grama de Pós-Graduação em Ecologia e Recursos Naturais, Universidade Federal de São Carlos PPGERN, UFScar. 158p.

Polkowska, Z.; Kozowska, K. Mazerska, Z.; Górecki, T. \& Namienik, J. 2003. Relationship between volatile organohalogen compounds in drinking water and human urine in Poland. Chemosphere. 53: 899-909.

Rörig, L.R. 2005. Usos Múltiplos e Qualidade das Águas da Bacia do Baixo Itajaí - Açu, SC: Elementos para Um Gerenciamento Integrado. Tese de Doutorado. Programa de Pós-Graduação em Ecologia e Recursos Naturais, Universidade Federal de São Carlos - PPGERN, UFScar. 295p.

Santos, C.L. 1987. Trialometanos: Resumo Atual. Engenharia Sanitária. 26: 190-194.

Shukla, V.K; Rastogi, A.N; Adukia, T.K; Raizada, R.B; Reddy, D.C.S. \& Singh, S., 2001. Organochlorine pesticides in carcinoma of the gallbladder: a casecontrol study. European Journal of Cancer Prevention. 10: 153-156.
Ströhlein GmbH \& Co. 1985. Coulomat 7020 CL: Operating Instructions Issue 10/96. Laboratory, Measurement and Environmental Technology. Kaarst, Alemanha: Ströhlein GmbH \& Co. 74p.

Tominaga, M.Y. \& Midio, A.F. 1999. Exposição humana a trialometanos presentes na água tratada. Rev. Saúde Pública. 33(4): 413-421.

Yamamoto, K., Fukushima, M. \& Kuroda, K. 1992. Total Organic Halogen: Chemical Pollution Parameter in Urban River Waters. Wat. Sci. Tech. 25 (11): 2532.

Submetido: Setembro/2007 Revisado: Abril/2008 Aceito: Fevereiro/2009 